\title{
熊果酸-3'-取代丙醇酯衍生物的合成及抗肿瘤活性研究
}

\author{
李 霞 ${ }^{a}$ 白 雪 ${ }^{a}$ 吴 科 ${ }^{a}$ 王裕军 ${ }^{a}$ 石万棋 ${ }^{b}$ \\ 李 颖*,a 尹述凡*,a \\ $\left({ }^{a}\right.$ 四川大学化学学院 成都 610064) \\ $\left({ }^{b}\right.$ 四川国康药业有限公司 成都 610041)
}

\begin{abstract}
摘要 熊果酸与 1,3-二溴丙烷反应制得中间体熊果酸-3'-溴代丙醇酯 $(\mathbf{1})$, 含澳醇酯 $\mathbf{1}$ 与含氮化合物反应, 合成了系列熊 果酸含氮衍生物 $\mathbf{2 a} \sim \mathbf{2} \mathbf{j}$, 均未见文献报道, 其结构经 ${ }^{1} \mathrm{H}$ NMR, ${ }^{13} \mathrm{C}$ NMR, IR, MS 确认. 初步的抗肿瘤生物活性研究表 明, 部分化合物的抗肿瘤活性与熊果酸相比均有提高, 其中化合物 $\mathbf{2}$ 具有更强的抑制活性.

关键词 熊果酸; 3'-溴代丙醇酯; 合成; 抗肿瘤
\end{abstract}

\section{Synthesis and Antitumor Activities of 3'-Substituted Propanolursolates}

\author{
$\mathrm{Li}_{\text {, Xia }}{ }^{a} \quad$ Bai, Xue $^{a} \quad \mathrm{Wu}, \mathrm{Ke}^{a} \quad$ Wang, Yujun $^{a} \quad$ Shi, Wangqi ${ }^{b} \quad$ Li, Ying ${ }^{*, a}$ \\ Yin, Shufan*,a \\ ( ${ }^{a}$ Collage of Chemistry, Sichuan University, Chengdu 610064) \\ ( ${ }^{b}$ Sichuan Guokang Pharmaceutical Co., Ltd, Chengdu 610041)
}

\begin{abstract}
Substituted propanolursolates (1) were obtained by a reaction of ursolic acid with trimethylene bromide. This intermediate reacted with several N-containing compounds to afford a series of new derivatives $\mathbf{2} \mathbf{a} \sim \mathbf{2} \mathbf{j}$. The structures of new products were characterized by means of ${ }^{1} \mathrm{H}$ NMR, ${ }^{13} \mathrm{C}$ NMR, IR and MS techniques. The preliminary bioassay test shows that several compounds give a good antitumor activity, and most of them are better than that of parent ursolic acid. Among them, compound $2 \mathrm{e}$ shows the best activity.
\end{abstract}

Keywords ursolic acid; 3'-substituted propanolursolate; synthesis; antitumor activity

熊果酸(UA)化学名 3 $\beta$-羟基-熊果-12-烯-28-酸, 是 一种广泛存在于自然界中的五环三萜化合物, 具有抗肝 炎、保肝、抗炎、抗艾滋病病毒、降血糖及抗肿瘤等丰 富的生物活性 ${ }^{[1]}$, 且副作用小、毒性低, 具有较大的临床 应用潜力. 特别是其抗炎 ${ }^{[2]}$ 、抗肿瘤活性及其作用机制 更是受到重视并开展了相关的研究 ${ }^{[3 \sim 7]}$. 研究表明, 熊 果酸不仅可以抵抗多种致肿瘤物、促肿瘤物, 而且可以 抑制多种恶性肿瘤细胞的生长, 有望成为低毒、高效的 新型抗肿瘤药物. 由于熊果酸自身作用强度较弱, 加上 其水溶性和脂溶性均较差, 生物利用率也低, 限制了其 在临床上的应用. 因此有必要对熊果酸的结构进行化学 修饰, 以期得到活性更高、毒性更小的新药. 本文在既 往研究的基础上, 通过在熊果酸结构中引入类氨基醇结 构的含氮分子片段, 合成了十个新型熊果酸-3'-取代丙
醇酯类衍生物(Scheme 1), 并对所得目标化合物进行了 NCI-H1975，MCF-7，SMMC-7721，HepG2，A549 和 SKOV3 细胞的抑制活性试验. 结果表明新化合物均具 有不同程度的抗肿瘤活性，且部分化合物抑制活性强于 熊果酸, 其中化合物 $\mathbf{2 e}$ 具有更强的抑制活性. 所合成的 十个新化合物均未见文献报道, 并经过 HRMS, IR, ${ }^{1} \mathrm{H}$ $\mathrm{NMR}$ 和 ${ }^{13} \mathrm{C} \mathrm{NMR}$ 表征确证其结构, 合成路线见 Scheme 1.

\section{1 实验部分}

\section{1 仪器与试剂}

IR 用 Perkin-Elmer 16PC-FT 型红外分光光度仪测定 $\left(\mathrm{KBr}\right.$ 压片); ${ }^{1} \mathrm{H}$ NMR, ${ }^{13} \mathrm{C}$ NMR 用 Bruker VA-400 磁共振

*E-mail: chuandayouji217@163.com

Received September 25, 2011; revised November 29, 2011; published online December 23, 2011. 


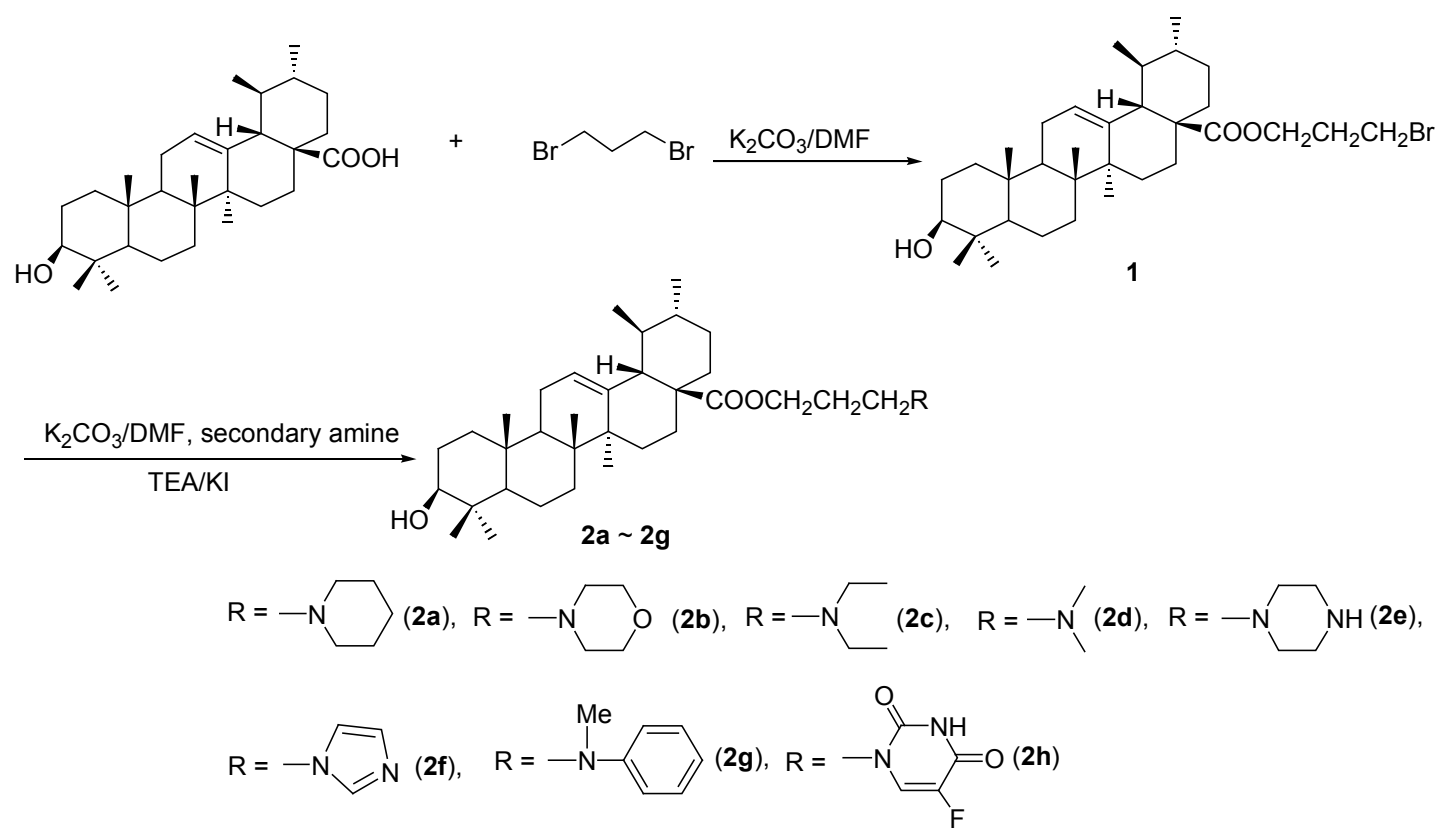

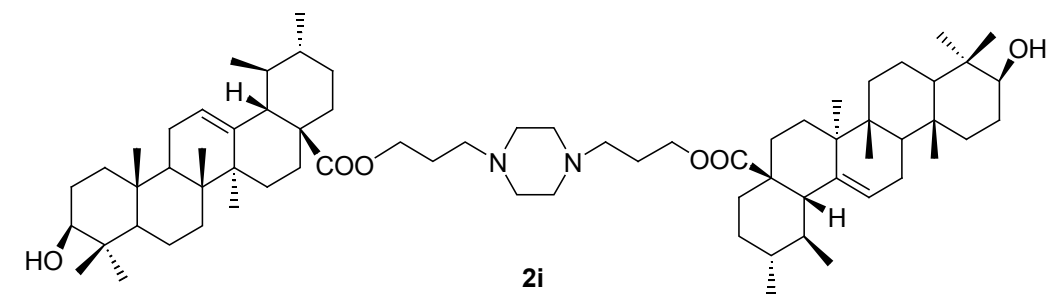

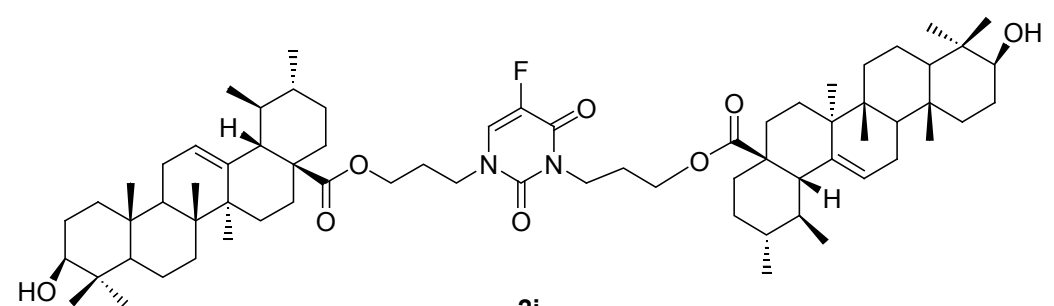

2j

Scheme 1

仪测定, TMS 作内标, $\mathrm{CDCl}_{3}$ 作溶剂; MS 用 Finnigan LCQDECA 和 Bruker Daltonics ESI-BioTOF Q(用内标法 测定高分辨质谱)测定; 熔点用 Yanaco 显微熔点测定仪 测定(温度计未经校正).

所用试剂和溶剂均为市售分析纯或化学纯, 除特别 注明外, 未进一步处理; 硅胶 $\mathrm{H}$ 和 $\mathrm{G}$ : 青岛海洋化工厂 生产, 未经活化. 熊果酸: 西安江兴生物科技有限公司 提供, 纯度为 $95 \%$ 或以上; 哌嗪: 天津市科密欧化学试 剂开发中心; $N$-甲基苯胺: 上海金山亭新化工试剂厂. 六氢吡啶, 吗啉: 成都科龙化工试剂厂; 咪唑, 5 -氟尿嘧 啶：爱斯特(成都)医药技术有限公司.

\section{2 合成}

\subsection{1 熊果酸 3'-溴代丙醇酯(1)的合成}

于 $100 \mathrm{~mL}$ 圆底烧瓶中依次加入熊果酸 $4.65 \mathrm{~g}$ (10 $\mathrm{mmol})$, 碳酸钾 $2.76 \mathrm{~g}(20 \mathrm{mmol})$, 碘化钾 $0.05 \mathrm{~g}, \mathrm{DMF}$ $40 \mathrm{~mL}, 4 \mathrm{~mL}$ 三乙胺, 1,3-二溴丙烷 $4.04 \mathrm{~g}(20 \mathrm{mmol}), 60$ ${ }^{\circ} \mathrm{C}$ 摚拌 $3.5 \mathrm{~h}$, 反应完毕后冷却至室温, 加入 $120 \mathrm{~mL}$ 水, 析出白色固体, 抽滤得到粗品, 柱层析纯化 $[V$ (石油 醚) $: V($ 乙酸乙酯 $)=4: 1$ ], 得到目标化合物 1 . 产率 $42 \%$, 白色固体, m.p. $78 \sim 79{ }^{\circ} \mathrm{C} ;{ }^{1} \mathrm{H}$ NMR $(400 \mathrm{MHz}$, $\left.\mathrm{CDCl}_{3}\right) \delta: 0.76\left(\mathrm{~s}, 3 \mathrm{H}, \mathrm{CH}_{3}\right), 0.78\left(\mathrm{~s}, 3 \mathrm{H}, \mathrm{CH}_{3}\right), 0.86(\mathrm{~d}$, $\left.J=6.4 \mathrm{~Hz}, 3 \mathrm{H}, \mathrm{CH}_{3}\right), 0.94\left(\mathrm{t}, J=6.2 \mathrm{~Hz}, 2-\mathrm{CH}_{3}\right), 0.99(\mathrm{~s}$, $\left.3 \mathrm{H}, \mathrm{CH}_{3}\right), 1.08\left(\mathrm{~s}, 3 \mathrm{H}, \mathrm{CH}_{3}\right), 1.21 \sim 1.72\left(\mathrm{~m}, 18 \mathrm{H}, 7 \mathrm{CH}_{2}+\right.$ $4 \mathrm{CH}), 1.73 \sim 1.83(\mathrm{~m}, 1 \mathrm{H}, \mathrm{H}-11), 1.92(\mathrm{dd}, J=3.5,8.8 \mathrm{~Hz}$, $\left.2 \mathrm{H}, \mathrm{CH}_{2}\right), 1.96 \sim 2.05(\mathrm{~m}, 1 \mathrm{H}, \mathrm{H}-11), 2.11 \sim 2.18(\mathrm{~m}, 2 \mathrm{H}$, $\mathrm{H}-22$ ), 2.21 (d, $J=11.48 \mathrm{~Hz}, 1 \mathrm{H}, \mathrm{H}-18), 3.21$ (dd, $J=5.1$, $10.9 \mathrm{~Hz}, 1 \mathrm{H}, \mathrm{H}-3), 3.46\left(\mathrm{t}, J=6.5 \mathrm{~Hz}, 2 \mathrm{H}, \mathrm{CH}_{2} \mathrm{Br}\right), 4.05 \sim$ $4.19\left(\mathrm{~m}, 2 \mathrm{H}, \mathrm{OCH}_{2}\right), 5.24$ (t, $\left.J=3.6 \mathrm{~Hz}, 1 \mathrm{H}, \mathrm{H}-12\right) ;{ }^{13} \mathrm{C}$ 
NMR (100 MHz, $\left.\mathrm{CDCl}_{3}\right) \delta: 177.34,138.28,125.65,79.02$, $61.80,55.23,52.94,48.21,39.08,36.76,28.15,24.25$; IR (KBr) $v$ : 3377, 2927, 2868, 1720, 1454, $1229 \mathrm{~cm}^{-1}$; HRMS (ESI) calcd for $\mathrm{C}_{33} \mathrm{H}_{53} \mathrm{BrO}_{3}[\mathrm{M}+\mathrm{H}]^{+}$577.3206, found 577.3381.

\subsection{2 熊果酸-3'-取代丙醇酯衍生物 $\mathbf{2} \mathbf{a} \sim 2 \mathrm{~g}$ 的合成}

于 $25 \mathrm{~mL}$ 圆底烧瓶中依次加入 $10.5 \mathrm{~g}(0.85 \mathrm{mmol})$, 碳酸钾 $0.35 \mathrm{~g}(2.55 \mathrm{mmol})$, 碘化钾少量, DMF $5 \mathrm{~mL}, 5$ 滴 三乙胺, 伯胺类化合物 $(1.7 \mathrm{mmol}), 60{ }^{\circ} \mathrm{C}$ 下搅拌反应, TCL 跟踪反应. 反应完毕后, 冷却至室温, 加入 $20 \mathrm{~mL}$ 水, 析出白色固体, 抽滤, 得到白色粗产物, 用硅胶柱 层析纯化, 得到目标化合物 2a, 同法制得 $\mathbf{2 b} \sim \mathbf{2 g}$.

2a: 白色絮状固体, 产率 73\%. m.p. 91 93 ${ }^{\circ} \mathrm{C} ;{ }^{1} \mathrm{H}$ NMR (400 MHz, $\left.\mathrm{CDCl}_{3}\right) \delta: 0.74\left(\mathrm{~s}, 3 \mathrm{H}, \mathrm{CH}_{3}\right) .0 .78(\mathrm{~s}, 3 \mathrm{H}$, $\left.\mathrm{CH}_{3}\right), 0.85$ (d, J=6.0 Hz, 3H, CH $\mathrm{CH}_{3}, 0.91$ (s, $3 \mathrm{H}, \mathrm{CH}_{3}$ ), 0.94 (s, 3H, $\left.\mathrm{CH}_{3}\right), 0.99$ (s, 3H, $\left.\mathrm{CH}_{3}\right), 1.08$ (s, 3H, $\mathrm{CH}_{3}$ ), $1.25 \sim 1.68\left(\mathrm{~m}, 30 \mathrm{H}, 13 \mathrm{CH}_{2}+4 \mathrm{CH}\right), 1.84 \sim 2.02(\mathrm{~m}, 6 \mathrm{H}$, $\left.3 \mathrm{NCH}_{2}\right), 2.21$ (d, $\left.J=11.43 \mathrm{~Hz}, 1 \mathrm{H}, \mathrm{H}-18\right), 3.21$ (dd, $J=$ $4.8,5.0 \mathrm{~Hz}, 1 \mathrm{H}, \mathrm{H}-3), 3.99 \sim 4.06\left(\mathrm{~m}, 2 \mathrm{H}, \mathrm{OCH}_{2}\right), 5.25(\mathrm{t}$, $J=3.6 \mathrm{~Hz}, 1 \mathrm{H}, \mathrm{H}-12) ;{ }^{13} \mathrm{C} \mathrm{NMR}\left(100 \mathrm{MHz}, \mathrm{CDCl}_{3}\right) \delta$ : 177.58, 138.24, 125.52, 78.93, 62.89, 55.23, 52.79, 49.40, $38.74,36.75,33.03,28.15,24.22$; IR (KBr) v: 3546, 2923, 2862, 2769, 1705, 1646, 1456, $1047 \mathrm{~cm}^{-1}$; HRMS (ESI) calcd for $\mathrm{C}_{38} \mathrm{H}_{63} \mathrm{O}_{3} \mathrm{~N}[\mathrm{M}+\mathrm{H}]^{+}$582.4816, found 582.4797 .

2b: 白色絮状固体, 产率 51\%. m.p. $80 \sim 82{ }^{\circ} \mathrm{C} ;{ }^{1} \mathrm{H}$ $\operatorname{NMR}\left(400 \mathrm{MHz}, \mathrm{CDCl}_{3}\right) \delta: 0.75\left(\mathrm{~s}, 3 \mathrm{H}, \mathrm{CH}_{3}\right), 0.78(\mathrm{~s}, 3 \mathrm{H}$, $\left.\mathrm{CH}_{3}\right), 0.86\left(\mathrm{~d}, J=6 \mathrm{~Hz}, 3 \mathrm{H}, \mathrm{CH}_{3}\right), 0.92\left(\mathrm{~s}, 3 \mathrm{H}, \mathrm{CH}_{3}\right), 0.94$ (s, $\left.3 \mathrm{H}, \mathrm{CH}_{3}\right), 0.99\left(\mathrm{~s}, 3 \mathrm{H}, \mathrm{CH}_{3}\right), 1.08\left(\mathrm{~s}, 3 \mathrm{H}, \mathrm{CH}_{3}\right), 1.22 \sim$ $1.78\left(\mathrm{~m}, 25 \mathrm{H}, 10 \mathrm{CH}_{2}+5 \mathrm{CH}\right), 2.21 \sim 2.46(\mathrm{~m}, 6 \mathrm{H}$, $\left.\left(\mathrm{CH}_{2}\right)_{3} \mathrm{~N}\right), 3.21(\mathrm{dd}, J=4.8,5.0 \mathrm{~Hz}, 1 \mathrm{H}, \mathrm{H}-3), 3.73(\mathrm{~s}, 4 \mathrm{H}$, $\left.\left(\mathrm{CH}_{2}\right)_{2} \mathrm{O}\right), 4.02\left(\mathrm{~d}, J=2.4 \mathrm{~Hz}, 2 \mathrm{H}, \mathrm{COOCH}_{2}\right), 5.30$ (t, $J=$ $3.6 \mathrm{~Hz}, 1 \mathrm{H}, \mathrm{H}-12) ;{ }^{13} \mathrm{C} \mathrm{NMR}\left(100 \mathrm{MHz}, \mathrm{CDCl}_{3}\right) \delta: 177.58$, $138.29,125.51,79.00,66.86,62.42,55.23,53.23,52.79$, $48.09,38.74,36.75,33.03,28.15,24.22$; IR (KBr) $v: 3431$, 2927, 2870, 1724, 1615, 1457, $1039 \mathrm{~cm}^{-1}$; HRMS (ESI) calcd for $\mathrm{C}_{37} \mathrm{H}_{61} \mathrm{O}_{4} \mathrm{~N}[\mathrm{M}+\mathrm{H}]^{+}$584.4609, found 584.4585 .

2c: 白色粉末固体, 产率 $55 \%$. m.p. $62 \sim 63{ }^{\circ} \mathrm{C} ;{ }^{1} \mathrm{H}$ $\operatorname{NMR}\left(400 \mathrm{MHz}, \mathrm{CDCl}_{3}\right) \delta: 0.75\left(\mathrm{~s}, 3 \mathrm{H}, \mathrm{CH}_{3}\right), 0.78(\mathrm{~s}, 3 \mathrm{H}$, $\left.\mathrm{CH}_{3}\right), 0.86\left(\mathrm{~d}, J=6 \mathrm{~Hz}, 3 \mathrm{H}, \mathrm{CH}_{3}\right), 0.92\left(\mathrm{~s}, 3 \mathrm{H}, \mathrm{CH}_{3}\right), 0.95$ (s, $\left.3 \mathrm{H}, \mathrm{CH}_{3}\right), 0.99$ (s, $\left.3 \mathrm{H}, \mathrm{CH}_{3}\right), 1.08\left(\mathrm{~s}, 3 \mathrm{H}, \mathrm{CH}_{3}\right), 1.20 \sim$ $1.79(\mathrm{~m}, 29 \mathrm{H}) 1.91\left(\mathrm{t}, J=4.0 \mathrm{~Hz}, 2 \mathrm{H}, \mathrm{CH}_{2}\right), 2.2 \sim 2.61(\mathrm{~m}$, $\left.6 \mathrm{H},\left(\mathrm{CH}_{2}\right)_{3} \mathrm{~N}\right), 3.21(\mathrm{dd}, J=4.8,5.0 \mathrm{~Hz}, 1 \mathrm{H}, \mathrm{H}-3), 4.03(\mathrm{~d}$, $\left.J=2.4 \mathrm{~Hz}, 2 \mathrm{H}, \mathrm{OCH}_{2}\right), 5.24(\mathrm{t}, J=3.6 \mathrm{~Hz}, 1 \mathrm{H}, \mathrm{H}-12) ;{ }^{13} \mathrm{C}$ NMR (100 MHz, $\left.\mathrm{CDCl}_{3}\right) \delta: 177.51,138.24,125.53,78.96$, $62.57,55.22,52.84,49.40,39.08,36.75,33.03,28.15$,
24.22; IR (KBr) v: 3431, 2927, 2870, 1724, 1457, 1039 $\mathrm{cm}^{-1}$; HRMS (ESI) calcd for $\mathrm{C}_{37} \mathrm{H}_{63} \mathrm{O}_{3} \mathrm{~N}[\mathrm{M}+\mathrm{H}]^{+}$: 570.4816, found 570.4755 .

2d: 白色絮状固体, 产率 42\%. m.p. 70 71 ${ }^{\circ} \mathrm{C} ;{ }^{1} \mathrm{H}$ NMR (400 MHz, $\left.\mathrm{CDCl}_{3}\right) \delta: 0.75\left(\mathrm{~s}, 3 \mathrm{H}, \mathrm{CH}_{3}\right), 0.78(\mathrm{~s}, 3 \mathrm{H}$, $\left.\mathrm{CH}_{3}\right), 0.86\left(\mathrm{~d}, J=15.2 \mathrm{~Hz}, 3 \mathrm{H}, \mathrm{CH}_{3}\right), 0.92\left(\mathrm{~s}, 3 \mathrm{H}, \mathrm{CH}_{3}\right)$, $0.94\left(\mathrm{~d}, J=11.9 \mathrm{~Hz}, 3 \mathrm{H}, \mathrm{CH}_{3}\right), 0.99$ (s, 3H, $\left.\mathrm{CH}_{3}\right), 1.08$ (s, $\left.3 \mathrm{H}, \mathrm{CH}_{3}\right), 1.21 \sim 1.83\left(\mathrm{~m}, 23 \mathrm{H}, 9 \mathrm{CH}_{2}+5 \mathrm{CH}\right), 1.91(\mathrm{t}, J=$ $\left.4.0 \mathrm{~Hz}, 2 \mathrm{H}, \mathrm{CH}_{2}\right), 2.25\left(\mathrm{~s}, 6 \mathrm{H}, \mathrm{NCH}_{3}\right), 2.29 \sim 2.42(\mathrm{~m}, 2 \mathrm{H}$, $\mathrm{NCH}_{2}$ ), 3.21 (dd, $\left.J=4.8,5.0 \mathrm{~Hz}, 1 \mathrm{H}, \mathrm{H}-3\right), 3.99 \sim 4.06(\mathrm{~m}$, $\left.2 \mathrm{H}, \mathrm{OCH}_{2}\right), 5.24(\mathrm{t}, J=3.6 \mathrm{~Hz}, 1 \mathrm{H}, \mathrm{H}-12) ;{ }^{13} \mathrm{C}$ NMR (100 $\left.\mathrm{MHz}, \mathrm{CDCl}_{3}\right) \delta: 177.52,138.26,125.52,78.95,62.54$, $55.23,52.84,48.21,39.08,36.72,28.15,24.22$; IR (KBr) v: 3432, 2927, 2867, 2767, 1724, 1460, $1042 \mathrm{~cm}^{-1}$; HRMS (ESI) calcd for $\mathrm{C}_{35} \mathrm{H}_{59} \mathrm{O}_{3} \mathrm{~N}[\mathrm{M}+\mathrm{H}]^{+}$542.4503, found 542.4506 .

2e: 白色絮状固体, 产率 57\%. m.p. 55 56 ${ }^{\circ} \mathrm{C} ;{ }^{1} \mathrm{H}$ NMR (400 MHz, $\left.\mathrm{CDCl}_{3}\right) \delta: 0.75\left(\mathrm{~s}, 3 \mathrm{H}, \mathrm{CH}_{3}\right), 0.78(\mathrm{~s}, 3 \mathrm{H}$, $\left.\mathrm{CH}_{3}\right), 0.85$ (d, $\left.J=6 \mathrm{~Hz}, 3 \mathrm{H}, \mathrm{CH}_{3}\right), 0.91$ (s, $\left.3 \mathrm{H}, \mathrm{CH}_{3}\right), 0.94$ (s, $\left.3 \mathrm{H}, \mathrm{CH}_{3}\right) 0.98$ (s, 3H, $\left.\mathrm{CH}_{3}\right), 1.07$ (s, $\left.3 \mathrm{H}, \mathrm{CH}_{3}\right), 1.18 \sim$ $1.85\left(\mathrm{~m}, 22 \mathrm{H}, 9 \mathrm{CH}_{2}+4 \mathrm{CH}\right), 1.91\left(\mathrm{t}, J=4.0 \mathrm{~Hz}, 2 \mathrm{H}, \mathrm{CH}_{2}\right)$, 2.21 (d, $J=11.43 \mathrm{~Hz}, 1 \mathrm{H}, \mathrm{H}-18), 2.41$ (s, 6H, $\mathrm{NCH}_{2}$ ), 2.89 (s, $4 \mathrm{H}, \mathrm{NHCH}_{2}$ ), 3.21 (dd, $J=4.8,5.0 \mathrm{~Hz}, 1 \mathrm{H}, \mathrm{H}-3$ ), $3.99 \sim 4.06\left(\mathrm{~m}, 2 \mathrm{H}, \mathrm{OCH}_{2}\right), 5.28(\mathrm{t}, J=3.6 \mathrm{~Hz}, 1 \mathrm{H}, \mathrm{H}-12)$; ${ }^{13} \mathrm{C}$ NMR $\left(100 \mathrm{MHz}, \mathrm{CDCl}_{3}\right) \delta: 177.52,138.22,125.49$, $78.70,62.58,55.23,52.84,48.21,47.51,39.08,36.72$, 28.15, 26.16; IR (KBr) v: 3325, 2924, 1722, 1591, 1455, $1140 \mathrm{~cm}^{-1}$; HRMS (ESI) calcd for $\mathrm{C}_{37} \mathrm{H}_{62} \mathrm{O}_{3} \mathrm{~N}_{2}[\mathrm{M}+\mathrm{H}]^{+}$ 583.4768, found 583.4796 .

2f: 白色絮状固体，产率 33\%. m.p.186 188 ${ }^{\circ} \mathrm{C} ;{ }^{1} \mathrm{H}$ NMR (400 MHz, $\left.\mathrm{CDCl}_{3}\right) \delta: 0.75\left(\mathrm{~s}, 3 \mathrm{H}, \mathrm{CH}_{3}\right), 0.78(\mathrm{~s}, 3 \mathrm{H}$, $\left.\mathrm{CH}_{3}\right), 0.85$ (d, $\left.J=6 \mathrm{~Hz}, 3 \mathrm{H}, \mathrm{CH}_{3}\right), 0.89$ (s, $\left.3 \mathrm{H}, \mathrm{CH}_{3}\right), 0.92$ (s, 3H, $\left.\mathrm{CH}_{3}\right), 1.04$ (s, 3H, $\left.\mathrm{CH}_{3}\right), 1.14$ (s, 3H, $\left.\mathrm{CH}_{3}\right), 1.91$ (t, $\left.J=4.0 \mathrm{~Hz}, 2 \mathrm{H}, \mathrm{CH}_{2}\right), 2.22\left(\mathrm{~d}, J=7.2 \mathrm{~Hz}, 2 \mathrm{H}, \mathrm{CH}_{2} \mathrm{~N}\right), 3.02$ (dd, $J=4.8,5.0 \mathrm{~Hz}, 1 \mathrm{H}, \mathrm{H}-3), 4.02(\mathrm{t}, J=2.4 \mathrm{~Hz}, 2 \mathrm{H}$, $\mathrm{OCH}_{2}$ ), 5.30 (t, $\left.J=3.6 \mathrm{~Hz}, 1 \mathrm{H}, \mathrm{H}-12\right), 6.78$ (s, $1 \mathrm{H}$, imidazole H-4), 7.22 (s, 1H, imidazole H-5), 7.96 (s, 1H, imidazole $\mathrm{H}-2) ;{ }^{13} \mathrm{C} \mathrm{NMR}\left(100 \mathrm{MHz}, \mathrm{CDCl}_{3}\right) \delta: 177.26,138.34$, $137.10,129.72,125.60,118.78,78.88,60.50,55.21,52.97$, $48.25,47.49,39.08,38.75,36.97,30.35,28.15,23.30$; IR (KBr) $v: 3381,3113,2924,2873,1714,1517,1457,1235$ $\mathrm{cm}^{-1}$; HRMS (ESI) calcd for $\mathrm{C}_{36} \mathrm{H}_{56} \mathrm{O}_{3} \mathrm{~N}_{2}[\mathrm{M}+\mathrm{H}]^{+}$ 565.4299, found 565.4307 .

2g: 白色絮状固体, 产率 42\%. m.p. 68 $69{ }^{\circ} \mathrm{C} ;{ }^{1} \mathrm{H}$ NMR (400 MHz, $\left.\mathrm{CDCl}_{3}\right) \delta: 0.74\left(\mathrm{~s}, 3 \mathrm{H}, \mathrm{CH}_{3}\right), 0.78(\mathrm{~s}, 3 \mathrm{H}$, 
$\left.\mathrm{CH}_{3}\right), 0.86$ (d, J=6 Hz, 3H, $\left.\mathrm{CH}_{3}\right), 0.92$ (s, 3H, $\left.\mathrm{CH}_{3}\right), 0.95$ (s, 3H, $\left.\mathrm{CH}_{3}\right), 0.99$ (s, 3H, $\left.\mathrm{CH}_{3}\right), 1.08\left(\mathrm{~s}, 3 \mathrm{H}, \mathrm{CH}_{3}\right), 1.19 \sim$ 1.99 (m, 25H), 2.93 (s, 3H, $\mathrm{NCH}_{3}$ ), 3.22 (dd, $J=4.8,5.0$ $\mathrm{Hz}, 1 \mathrm{H}, \mathrm{H}-3), 3.46\left(\mathrm{~s}, 2 \mathrm{H}, \mathrm{CH}_{2} \mathrm{~N}\right), 4.06$ (t, $J=2.4 \mathrm{~Hz}, 2 \mathrm{H}$, $\mathrm{OCH}_{2}$ ), 5.23 (t, $\left.J=3.6 \mathrm{~Hz}, 1 \mathrm{H}, \mathrm{H}-12\right), 6.71$ (s, $\left.2 \mathrm{H}, \alpha-\mathrm{ArH}\right)$, $6.73(\mathrm{~s}, 1 \mathrm{H}, \gamma-\mathrm{ArH}), 7.23(\mathrm{dd}, J=8.0,15.2 \mathrm{~Hz}, 2 \mathrm{H}$, $\beta$-ArH); ${ }^{13} \mathrm{C}$ NMR $\left(100 \mathrm{MHz}, \mathrm{CDCl}_{3}\right) \delta: 177.55,149.12$, $138.21,129.21,125.63,116.35,112.28,78.99,61.98$, $55.21,52.94,48.21,47.55,39.08,38.75,36.97,30.35$, 28.15, 23.30, 17.12; IR (KBr) v: 3437, 2925, 2869, 1723, $1600,1506,1453,1140 \mathrm{~cm}^{-1}$; HRMS (ESI) calcd for $\mathrm{C}_{40} \mathrm{H}_{61} \mathrm{O}_{3} \mathrm{~N}[\mathrm{M}+\mathrm{H}]^{+}$604.4659, found 604.4695.

\subsection{3 熊果酸-3'-取代丙醇酯衍生物 $2 \mathrm{~h}$ 的合成}

于 $25 \mathrm{~mL}$ 圆底烧瓶中依次加入化合物 $\mathbf{1} 0.5 \mathrm{~g}(0.85$ $\mathrm{mmol}$ ), 碘化钾少量, DMF $5 \mathrm{~mL}, 1 \mathrm{~mL}$ 三乙胺, 氟尿嘧 啶 $0.25 \mathrm{~g}(1.7 \mathrm{mmol})$, 室温下摚拌反应, TCL 跟踪反应. 反应完毕后, 加入 $20 \mathrm{~mL}$ 双蒸水, 析出白色固体, 抽滤, 得到白色粗产物, 用硅胶柱层析纯化 [ $V$ (石油醚) : $V($ 乙 酸乙酯) $=1: 1]$, 得到目标化合物 $\mathbf{2 h}$.

2h: 白色固体，产率 $22 \%$. m.p. $133 \sim 135{ }^{\circ} \mathrm{C} ;{ }^{1} \mathrm{H}$ NMR (400 MHz, $\left.\mathrm{CDCl}_{3}\right) \delta: 0.74\left(\mathrm{~d}, J=12 \mathrm{~Hz}, 3 \mathrm{H}, \mathrm{CH}_{3}\right)$, 0.78 (s, 3H, $\left.\mathrm{CH}_{3}\right), 0.86$ (d, $\left.J=6 \mathrm{~Hz}, 3 \mathrm{H}, \mathrm{CH}_{3}\right), 0.91$ (s, 3H, $\mathrm{CH}_{3}$ ), 0.95 (s, 3H, $\mathrm{CH}_{3}$ ), 0.99 (s, 3H, $\mathrm{CH}_{3}$ ), 1.07 (s, 3H, $\left.\mathrm{CH}_{3}\right), 2.01\left(\mathrm{t}, J=4.8 \mathrm{~Hz}, 2 \mathrm{H}, \mathrm{CH}_{2} \mathrm{~N}\right), 3.21$ (d, $J=4.8,5.0$ $\mathrm{Hz}, 1 \mathrm{H}, \mathrm{H}-3), 4.06$ (t, $\left.J=2.4 \mathrm{~Hz}, 2 \mathrm{H}, \mathrm{OCH}_{2}\right), 5.26(\mathrm{t}, J=$ $3.6 \mathrm{~Hz}, 1 \mathrm{H}, \mathrm{H}-12), 7.27(\mathrm{~d}, J=2 \mathrm{~Hz}, 1 \mathrm{H}, \mathrm{NCH}=\mathrm{CF})$, $9.58 \sim 9.62(\mathrm{~m}, 1 \mathrm{H}, \mathrm{NH}) ;{ }^{13} \mathrm{C} \mathrm{NMR}\left(100 \mathrm{MHz}, \mathrm{CDCl}_{3}\right) \delta$ : $177.38,157.16,149.61,141.12,138.25,128.88,125.61$, $78.92,61.85,55.21,52.84,48.21,47.55,39.08,38.73$, $36.59,30.59,28.15$; IR (KBr) v: 3443, 3073, 2925, 2866, 1719, 1456, 1238, $1140 \mathrm{~cm}^{-1}$; HRMS (ESI) calcd for $\mathrm{C}_{37} \mathrm{H}_{55} \mathrm{O}_{5} \mathrm{~N}_{2} \mathrm{~F}[\mathrm{M}+\mathrm{H}]^{+}$627.4103, found 627.4167.

\subsection{4 熊果酸-3'-取代丙醇酯衍生物 $2 \mathbf{i}, 2 \mathbf{j}$ 的合成}

于 $25 \mathrm{~mL}$ 圆底烧瓶中依次加入化合物 $11.0 \mathrm{~g}(1.7$ $\mathrm{mmol})$, 碳酸钾 $0.50 \mathrm{~g}(5.1 \mathrm{mmol})$, 碘化钾少量, DMF 10 $\mathrm{mL}, 10$ 滴三乙胺, 哌嗪 $0.075 \mathrm{~g}(0.85 \mathrm{mmol})$ 或氟尿嘧啶 $0.125 \mathrm{~g}(0.85 \mathrm{mmol}), 60{ }^{\circ} \mathrm{C}$ 下搅拌反应, TCL 跟踪反应. 反应完毕后, 冷却至室温, 加入 $20 \mathrm{~mL}$ 双蒸水, 析出白 色固体, 抽滤, 得到白色粗产物, 用硅胶柱层析纯化 $[V$ (石油醚) $: V($ 乙酸乙酯 $)=1: 3]$, 得到目标化合物 $\mathbf{2 i}$ 或 $\mathbf{2 j}$.

2i: 白色絮状固体, 产率 50.6\%. m.p. 82 84 ${ }^{\circ} \mathrm{C} ;{ }^{1} \mathrm{H}$ NMR (400 MHz, $\left.\mathrm{CDCl}_{3}\right) \delta: 0.74\left(\mathrm{~d}, J=12 \mathrm{~Hz}, 6 \mathrm{H}, \mathrm{CH}_{3}\right)$, 0.78 (s, 6H, $\left.\mathrm{CH}_{3}\right), 0.85$ (d, $\left.J=6 \mathrm{~Hz}, 6 \mathrm{H}, \mathrm{CH}_{3}\right), 0.91$ (s, 6H, $\left.\mathrm{CH}_{3}\right), 0.95$ (s, 6H, $\left.\mathrm{CH}_{3}\right), 0.99$ (s, 6H, $\left.\mathrm{CH}_{3}\right), 1.08$ (s, 6H,
$\left.\mathrm{CH}_{3}\right), 2.20 \sim 2.42\left(\mathrm{~m}, 12 \mathrm{H},\left(\mathrm{CH}_{2}\right)_{3} \mathrm{~N}\right), 3.21(\mathrm{~d}, J=4.8,5.0$ $\mathrm{Hz}, 1 \mathrm{H}, \mathrm{H}-3), 4.02$ (d, $\left.J=2.4 \mathrm{~Hz}, 4 \mathrm{H}, \mathrm{OCH}_{2}\right), 5.23$ (t, $J=$ $3.6 \mathrm{~Hz}, 2 \mathrm{H}, \mathrm{H}-12) ;{ }^{13} \mathrm{C} \mathrm{NMR}\left(100 \mathrm{MHz}, \mathrm{CDCl}_{3}\right) \delta: 177.52$, $138.22,125.49,78.70,62.58,55.23,52.84,48.21,47.51$, $39.08,36.72,28.15,26.16$; IR (KBr) $v: 3449,2925,2865$, $1724,1458,1038 \mathrm{~cm}^{-1}$; HRMS (ESI) calcd for $\mathrm{C}_{72} \mathrm{H}_{114^{-}}$ $\mathrm{O}_{6} \mathrm{~N}_{2}[\mathrm{M}+\mathrm{H}]^{+}$1079.8693, found 1079.8727.

2j: 白色絮状固体，产率 $42 \%$. m.p. $147 \sim 150{ }^{\circ} \mathrm{C}$; ${ }^{1} \mathrm{H}$ NMR $\left(400 \mathrm{MHz}, \mathrm{CDCl}_{3}\right) \delta: 0.74(\mathrm{~s}, J=12 \mathrm{~Hz}, 6 \mathrm{H}$, $\left.\mathrm{CH}_{3}\right), 0.78\left(\mathrm{~s}, 6 \mathrm{H}, \mathrm{CH}_{3}\right), 0.86\left(\mathrm{~d}, J=6 \mathrm{~Hz}, 6 \mathrm{H}, \mathrm{CH}_{3}\right), 0.91$ (s, 6H, $\left.\mathrm{CH}_{3}\right), 0.95$ (s, 6H, $\left.\mathrm{CH}_{3}\right), 0.98$ (s, 6H, $\left.\mathrm{CH}_{3}\right), 1.08$ (s, $\left.6 \mathrm{H}, \mathrm{CH}_{3}\right), 1.91$ (t, $\left.J=4.0 \mathrm{~Hz}, 4 \mathrm{H}, \mathrm{CH}_{2}\right), 2.21(\mathrm{t}, J=4.8 \mathrm{~Hz}$, $\left.4 \mathrm{H}, \mathrm{CH}_{2} \mathrm{~N}\right), 3.22(\mathrm{~d}, J=4.8,5.0 \mathrm{~Hz}, 1 \mathrm{H}, \mathrm{H}-3), 4.06(\mathrm{~m}$, $\left.J=2.4 \mathrm{~Hz}, 4 \mathrm{H}, \mathrm{OCH}_{2}\right), 5.24$ (t, $\left.J=3.6 \mathrm{~Hz}, 1 \mathrm{H}, \mathrm{H}-12\right), 7.24$ $(\mathrm{d}, J=4.8 \mathrm{~Hz}, 2 \mathrm{H}, \mathrm{NCH}=\mathrm{CF}) ;{ }^{13} \mathrm{C}$ NMR $(100 \mathrm{MHz}$, $\mathrm{CDCl}_{3}$ ) $\delta: 177.38,157.16,149.61,141.12,138.25,128.88$, $125.61,78.92,61.85,55.21,52.84,48.21,47.55,39.08$, $38.73,36.59,30.59,28.15$; IR (KBr) v: 3521, 2927, 2870, 1719, 1662, 1461, 1373, 1231, $1040 \mathrm{~cm}^{-1}$; HRMS (ESI) calcd for $\mathrm{C}_{70} \mathrm{H}_{107} \mathrm{O}_{8} \mathrm{~N}_{2} \mathrm{~F}[\mathrm{M}+\mathrm{Na}]^{+}$1145.7888, found 1145.7867.

\subsection{4 抗肿瘤活性实验}

将生长良好的人肺癌细胞 A549、人卵巢癌 SKOV3 细胞、人肝癌细胞 Hep G2 分别接种于 96 孔板, $2 \times 10^{3}$ / 孔. $24 \mathrm{~h}$ 后将不同浓度的试药分别加入板中, $100 \mu \mathrm{L} /$ 孔， 培养 $48 \mathrm{~h}$. 然后加入 MTT 溶液 $10 \mu \mathrm{L}(5 \mathrm{mg} / \mathrm{mL}), 37{ }^{\circ} \mathrm{C}$ 作用 $4 \mathrm{~h}$, 弃上清, 加入 DMSO $150 \mu \mathrm{L} /$ 孔, 室温振摇 30 min, 测定 OD570 $\mathrm{nm}$ 值. 根据细胞增殖抑制率公式计 算：抑制率 $(\%)=($ 对照组 $\mathrm{OD}$ 值一实验组 $\mathrm{OD}$ 值) $/$ 对照组 $\mathrm{OD}$ 值 $\times 100 \% . \mathrm{IC}_{50}$ 值利用统计软件进行计算. 实验结 果见表 1 .

\section{2 结果与讨论}

\section{1 合成}

利用熊果酸在易溶的 DMF 中, 以碳酸钾做碱，三 乙胺作缚酸剂, 碘化钾作催化剂, 与两倍量的 1,3 -二溴 丙烷反应得到中间体熊果酸-3'-溴丙醇酯. 为防止发生 双取代，卤代烃需过量. 对于 $\mathbf{2 a} \sim \mathbf{2 j}$, 可用不同类型的 氨基物或含氮杂环进行亲核取代得到目标化合物，反应 可通过控制氨基物或含氮杂环与中间体 $\mathbf{1}$ 的量的比例分 别得到单取代和双取代的化合物.

\section{2 化合物结构表征}

\subsection{1 ${ }^{1} \mathrm{H}$ NMR 谱图}

所合成的化合物中, 熊果酸 C-12 的化学位移出现 
表 1 目标化合物对肿瘤细胞的抑制活性 ${ }^{a}$

Table 1 Antitumor activities for target compounds

\begin{tabular}{|c|c|c|c|c|c|c|}
\hline \multirow{2}{*}{ Compd. } & \multicolumn{6}{|c|}{$\mathrm{IC}_{50} /\left(\mu \mathrm{mol} \cdot \mathrm{L}^{-1}\right)$} \\
\hline & 人肺癌 A549 & 人卵巢癌 SKOV3 & 人肝癌 SMMC-7721 & 人肺癌 NCI-H1975 & 人肝癌 Hep G2 & 乳腺癌 MCF-7 \\
\hline UA & 45.8 & 46.4 & $>40$ & $>40$ & 28.5 & 34.1 \\
\hline 1 & 52.8 & 44.1 & $>40$ & 34.4 & 33.3 & 30.4 \\
\hline $2 \mathbf{a}$ & 34.4 & 38.7 & $>40$ & 24.5 & 26.5 & 26.1 \\
\hline $2 b$ & 32.3 & 30.2 & $>40$ & $>40$ & 24.6 & 26.5 \\
\hline $2 \mathrm{c}$ & 35.6 & 28.9 & $>40$ & 30.5 & 30.3 & 25.1 \\
\hline 2d & 51.5 & 39.6 & $>40$ & 38.2 & 28.1 & 34.3 \\
\hline $2 \mathrm{e}$ & 27.4 & 25.1 & $>40$ & 28.2 & 18.8 & 19.0 \\
\hline $2 f$ & $>20$ & $>20$ & $>20$ & $>40$ & 29.4 & 19.5 \\
\hline $2 \mathrm{~g}$ & 61.6 & 45.7 & $>40$ & $>40$ & 48.6 & 47.8 \\
\hline $2 \mathrm{~h}$ & 75.3 & 79 & $>40$ & $>40$ & 72.9 & 60.9 \\
\hline $2 \mathbf{i}$ & $>40$ & $>40$ & $>40$ & $>40$ & $>40$ & $>40$ \\
\hline $2 \mathbf{j}$ & $>80$ & $>80$ & $>40$ & $>40$ & $>80$ & $>80$ \\
\hline
\end{tabular}

$a$ 空白组作为对照组

在 $\delta 5.24$ 附近; 熊果酸 C-3 位质子的化学位移出现在 $\delta$ 3.21 附近, 熊果酸多个甲基的化学位移出现在 $\delta 0.69 \sim$ 1.20 范围内, 熊果酸 C-28 位由于酯基中氧的诱导效应 使亚甲基的化学位移出现在 $\delta 4.08$ 附近; 所合成的化合 物 $\mathbf{1}$ 中, $\delta 3.46$ 为 $\mathrm{CH}_{2} \mathrm{Br}$ 的化学位移; 化合物 $\mathbf{2 a} \sim \mathbf{2 e}$ 中, 出现在 $\delta 5.23$ 左右的质子化学位移是熊果酸的 $\mathrm{H}-12$; 在 $\delta 4.01$ 左右出现的质子化学位移是 $\mathrm{OCH}_{2}$ 的; 在 $\delta 3.22$ 附近出现的质子化学位移是熊果酸的 H-3; $2 \mathbf{a}$ 中在 $\delta$ 2.33 附近的化学位移为 $\mathrm{N}\left(\mathrm{CH}_{3}\right)_{2}$ 的; $\mathbf{2 b} \sim \mathbf{2 d}, \mathbf{2 e}$ 中 $\delta$ $2.23 \sim 2.47$ 附近出现的质子化学位移是 $\mathrm{N}\left(\mathrm{CH}_{2}\right)_{2} ; \mathbf{2 d}, \mathbf{2 e}$ 分别在 $\delta 3.73$ 和 2.25 附近出现了质子化学位移分别是 $\mathrm{O}\left(\mathrm{CH}_{2}\right)_{2}$ 和 $\mathrm{HN}\left(\mathrm{CH}_{2}\right)_{2} ; \delta 1.10 \sim 2.10$ 范围内出现的是熊果 酸的多个亚甲基和次甲基质子化学位移; $\delta \quad 0.70 \sim 1.08$ 范围内是熊果酸的多个甲基质子化学位移. 化合物 $\mathbf{2 f} \sim$ $\mathbf{2 j}$ 中, 除了含有 $\mathbf{2 a} \sim 2 \mathrm{e}$ 中熊果酸结构中的特征质子化 学位移, 还有各自芳香环上的特征质子化学位移, 如 $2 \mathbf{f}$ 中 $\delta 6.89,7.15,7.59$ 就是咪唑环上 3 个 $\mathrm{H}$ 的质子化学位 移; $\mathbf{2} \mathbf{j}$ 中 $\delta 7.25$ 为氟尿嘧啶环上的 $\mathrm{CH}$ 的质子化学位移.

\subsection{2 ${ }^{13} \mathrm{C}$ NMR 谱图}

在所合成的化合物中均有熊果酸结构的特征化学 位移, $\delta 177.50$ 附近的化学位移为 $\mathrm{COOH}$ 的, 在 $\delta 138$ 附 近的化学位移为 C-13, 在 $\delta 125$ 附近的化学位移为 C-12, 在 $\delta 78.90$ 附近的化学位移为 C-3, 在 $\delta 61.80$ 附近的化 学位移为 $\mathrm{OCH}_{2}$ 的; $\mathbf{2 a} \sim \mathbf{2 j}$ 中的 $\mathrm{CH}_{2} \mathrm{~N}$ 的的化学位移出 现在 $\delta 47.50$ 附近. 每个化合物均有自己的特征化学位 移. 如 $2 \mathbf{d}$ 中在 $\delta 66.82$ 出现的化学位移为吗啉环 $\mathrm{O}(\mathrm{CH})_{2}$; 2f 中在 $\delta 118.78,129.72,137.10$ 出现的化学位移为咪唑 环上的 3 个 C; $2 \mathrm{~g}$ 中在 $\delta 112.28,116.30,125.66,129.21$,
$138.23,149.20$ 出现的化学位移为苯环上的 6 个 C.

\subsubsection{IR 谱图}

所合成的化合物 $\mathbf{2 a} \sim 2 \mathrm{e}$ 中, $3440 \sim 3420 \mathrm{~cm}^{-1}$ 附近 出现 $\mathrm{OH}$ 的伸缩振动吸收峰, 在 $3100 \mathrm{~cm}^{-1}$ 范围内出现 $\mathrm{C}=\mathrm{N}$ 的伸缩振动吸收峰, 在 $2860 \sim 2920 \mathrm{~cm}^{-1}$ 左右区域 系甲基、亚甲基、次甲基碳氢键伸缩振动吸收峰，在 $1710 \sim 1724 \mathrm{~cm}^{-1}$ 范围内出现羰基 $\mathrm{C}=\mathrm{O}$ 的中等强度吸 收峰, 在 $1640 \mathrm{~cm}^{-1}$ 范围内出现一 $\mathrm{NH}$ 的弯曲振动吸收 峰, 在 $1600 \sim 1500 \mathrm{~cm}^{-1}$ 范围内出现苯环骨架的吸收峰.

\subsubsection{MS 谱图}

目标化合物 2a 2e 质子峰都以所预想的准分子离 子峰 $[\mathrm{M}+\mathrm{H}]^{+}$的形式出现, 说明该类化合物均比较稳定, HRMS 检测结果与目标化合物的分子组成一致.

\section{3 抗肿瘤活性实验}

表 1 显示, 化合物 $2 \mathrm{a}, 2 \mathrm{~b}, 2 \mathrm{c}, 2 \mathrm{~d}$ 和 $2 \mathrm{e}$ 对人肺癌 A549、人卵巢癌 SKOV3、人肝癌 SMMC-7721、人肺癌 NCI-H1975、人肝癌 Hep G2 和人乳腺癌 MCF-7 细胞的 抑制活性较熊果酸均有所增强，其中化合物 $2 \mathrm{e}$ 的抑制 活性最高. 化合物 $2 \mathrm{f}, 2 \mathrm{~g}, 2 \mathrm{~h}, \mathbf{2 i}$ 和 $\mathbf{2 j}$ 的抑制活性均没有 熊果酸强. 由实验结果显示，分子量较小的化合物 $\mathbf{2 a} \sim$ 2e 的生物活性比分子量较大的化合物 $\mathbf{2 f} \sim \mathbf{2 j}$ 的抑制活 性高, 结构中含有二级未饱和氮(化合物 2e)的抑制活性 比其他化合物的抑制活性高，脂肪族或脂肪环族含氮结 构比芳香族、杂环抑制活性高. 进一步深入研究正在进 行中.

致谢 本文中的抗肿瘤活性实验由四川大学华西药学 院药理教研室包旭老师等完成, 所有谱图和相关数据由 
四川大学分析测试中心提供, 在此表示衷心的感谢.

\section{References}

[1] Song, X.-K.; Wu, L.-J. Textbook of Natural Medicinal Chemistry, Chemical Industry Press, Beijing, 2004, pp. 198 200 (in Chinese). (宋晓凯, 吴立军, 天然药物化学, 化学工业出版社, 北京, 2004, pp. 198 200.)

[2] Yang, D.-J.; Li, Y.; Yin, S.-F. Chin. J. Org. Chem. 2008, 28, 1055 (in Chinese).

(杨定菊, 李颖, 尹述凡, 有机化学, 2008, 28, 1055.)

[3] Huang, J.; Sun, Y.; Lu, S.-X.; Su, T.; Jiao, S.-C. Chin. J. Integr. Trad. Western Med. 1999, 19, 296 (in Chinese).

(黄镜，孙燕，陆士新，苏涛，焦顺昌，中国中西医结合杂志， 1999, 19, 296.)
[4] Barry, M. A.; Behnek, C. A.; Eastman, A. Biochem. Pharmacol. 1990, 40, 2353.

[5] Zhang, Q.-P.; Xie, L.-K.; Deng, T.; Hu, Z.-F.; Chen, H.-M.; Tan, J.-Q. Basic Clinics Med. 2004, 24, 414 (in Chinese).

(张秋萍, 谢路琨, 邓涛, 胡志芳, 陈会敏, 谭锦泉, 基础医学与 临床, 2004, 24, 414.)

[6] Li, J.; Xu, L.-Z.; Zhu, W.-P.; Zhang, T.-M.; Li, X.-M.; Jin, A.-P.; Huang, K.-M.; Li, D.-L.; Yang, Q.-Y. China Oncol. 1999, 9, 395 (in Chinese).

(李杰, 许良中, 朱伟萍, 张太明, 李小妹, 金爱萍, 黄抗美, 李 栋良, 杨庆尧, 中国癌症杂志, 1999, 9, 395.)

[7] Knighton, D. R.; Silver, I. A.; Hunt, T. K. Surgery 1981, 90.261.

[8] Wu, Q.-N.; Huang, W.; Huang, M.-S. J. Oncol. 2004, 10, 145 (in Chinese).

(吴其年, 黄炜, 黄敏珊, 肿瘤学杂志, 2004, 10, 145.)

(Lu, Y.) 